\title{
Costos de los egresos inoportunos de origen no médico en una clínica de alto nivel de complejidad en Colombia
}

\section{The costs of nonclinical delayed discharge in a tertiary care clinic in Colombia}

\author{
Alfredo Hernández-Ruiz, Didier Rave-Zuluaga, Francisco Fabián Jiménez-Quiroz, \\ Luisa Fernanda Rubio-Ayala • Rionegro (Colombia)
}

DOI: https://doi.org/10.36104/amc.2020.1255

\section{Resumen}

Objetivo: describir los pacientes con egreso inoportuno de origen no médico, sus características sociodemográficas, clínicas y estimar los costos derivados, durante el 01 de junio y el 31 de agosto de 2017, en una institución de salud de alto nivel de complejidad.

Material y métodos: estudio observacional, retrospectivo. La definición operativa de egreso inoportuno correspondía a pacientes que desde el punto de vista clínico podían ser dados de alta, pero continuaban ocupando una cama por problemas no médicos. Los datos fueron tomados de las historias clínicas y para el análisis de costo, se tuvieron en cuenta el total de los costos generados por la estancia inapropiada, incluyendo el costo día de hospitalización, insumos, medicamentos y evaluaciones médicas. La perspectiva del costo fue desde el pagador.

Resultados: de los 3273 egresos durante el periodo analizado, se presentaron 79 casos de egresos inoportunos (2.4\%). La edad media de los pacientes fue de 49 años. El $62 \%$ eran del régimen subsidiado; $60.7 \%$ eran procedentes de otros departamentos o municipios lejanos. El total de días hospitalización perdidos por esta causa fue de 547, con una media de 6.6 días por paciente. Los servicios más afectados fueron medicina interna y cuidados paliativos. Las principales causas fueron: necesidad de tiquetes para retornar al lugar de procedencia, alta temprana por medicamentos o insumos para el egreso y necesidad de oxígeno. El costo total que se generó por egreso inoportuno fue US\$83 935, con un valor promedio de US\$1062.47 por paciente.

Conclusión: el egreso inoportuno es un problema evidente en la atención. Las principales causas que encontramos fueron problemas sociales y traslados regionales. Además genera un gasto adicional importante de recursos materiales y económicos tanto para las instituciones como para el sistema. (Acta Med Colomb 2020; 45. DOI: https://doi.org/10.36104/amc.2020.1255).

Palabras clave: utilización inadecuada de la hospitalización, egreso inoportuno, costos.

\section{Abastract}

Objective: to describe the sociodemographic and clinical characteristics of patients with nonclinical delayed discharge and estimate the resulting costs in a tertiary healthcare facility from June 1 to August 31, 2017.

Materials and methods: a retrospective observational study. The working definition of delayed discharge was patients who, from a clinical perspective, could be discharged but who continued to occupy a bed due to nonclinical problems. The data were taken from the medical charts, and the total costs incurred by the inappropriate length of stay were used for the cost analysis, including the cost per inpatient day, supplies, medications and medical assessments, from the payer's cost perspective.

Results: of the 3,273 discharges during the study period, there were 79 cases of delayed discharge $(2.4 \%)$. The mean age of the patients was 49 years. Sixty-two percent had subsidized medical insurance; $60.7 \%$ came from other departments or distant towns. The total number of inpatient days lost due to this cause was 547 , with a me of 6.6 days per patient. The departments which were most
Dr. Alfredo Hernández-Ruiz: Médico Internista, MSc Epidemiología Clínica, Unidad de Investigación; Dr. Didier Rave-Zuluaga: Médico Auditor, Unidad de Auditoría Médica; Dres. Francisco Fabián Jiménez-Quiroz y Luisa Fernanda Rubio-Ayala: Médicos Generales, Unidad de Hospitalización. Clínica Somer. Rionegro (Colombia).

Correspondencia: Dr. Alfredo Hernández-Ruiz. Rionegro (Colombia).

E-mail: alfredohdezmd@hotmail.com

Recibido: 15/V/2020 Aceptado: 17/VII/2020 
affected were internal medicine and palliative care. The main causes were: the need for tickets to return to their place of origin, early discharge due to medications or discharge supplies and the need for oxygen. The total cost incurred due to delayed discharge was USD 83,935, with an average of USD 1,062.47 per patient.

Conclusion: delayed discharge is an evident healthcare problem. The main causes found in this study were social and regional transport issues. Delayed discharge also causes a significant additional expense in material and financial resources for both the institutions and the system. (Acta

Med Colomb 2020; 45. DOI: https://doi.org/10.36104/amc.2020.1255).

Key words: inappropriate use of hospitalization, delayed discharge, costs.

\section{Introducción}

La optimización de las camas en una institución de salud es un proceso que facilita el uso adecuado de los recursos, lo cual se convierte en una política no sólo institucional, sino también gubernamental (1). A pesar de esto, trabajos realizados en diferentes países, muestran que estos procesos muchas veces no son eficientes, lo que conlleva a estancias inapropiadas, generando así desperdicio de recursos tanto económico como humano y exponen a los pacientes a riesgos innecesarios, como infecciones, caídas, trombosis, aumento de costos, entre otros $(2,3)$. Además con el desbalance entre la oferta y demanda de las camas, si el paciente hospitalizado tiene inconveniente para irse, no libera una cama para ingresar un paciente procedente de otras áreas como urgencias, cirugía, entre otros, colapsando estos servicios (4).

Adicionalmente los procesos más sensibles que afectan el uso de las camas de una institución son la disponibilidad de la cama, el inicio oportuno del tratamiento y el egreso del paciente (5). Este último ha cobrado gran relevancia en la actualidad; si bien en nuestro país se han sumado esfuerzos para hacerlo más eficiente, reflejado en estrategias como el traslado a programas domiciliarios, entrega ágil de medicamentos para su egreso, programas especiales (diabetes, anticoagulación, oxígenoterapia, cáncer, entre otros), rondas diarias, auditorías (auditoría en tiempo real), guías nacionales (6), vemos que el impacto en muchas ocasiones es bajo, llevando a estancias innecesaria (7), que incluso pueden afectar más de $30 \%$ de los pacientes hospitalizados $(8,9)$.

Una estancia inapropiada puede estar relacionada con un egreso no oportuno, llamado también alta diferida del inglés «delayed discharge». Este concepto que se resume como bloqueo de una cama hospitalaria, es definida por The National Health Service (NHS) de Inglaterra, cuando un paciente desde el punto de vista clínico puede ser dado de alta, pero continúa ocupando una cama (10), concepto también aceptado en países latinoamericanos (11). El egreso inoportuno es un problema de alto impacto, se considera que puede explicar incluso el $70 \%$ de las causas de estancia inapropiada (12). Además generar alta perdida de recurso; reportes de países como Inglaterra anotan que puede llegar a costar cerca de $€ 100$ millones y consumir 1.2 millones de días-cama anualmente (13).
El egreso inoportuno de origen no médico generalmente está dado por las condiciones o necesidad que tienen los pacientes para su egreso. Estas condiciones pueden variar dependiendo de los países, sistemas de salud, condición clínica o edad. En España, Giraldo y cols. (11) describen el problema en un servicio de medicina interna, encontrando como causa principal las dificultades sociofamiliares para hacerse cargo de los pacientes al egreso. Hendy y cols. en el Reino Unido (14), describen luego de analizar los posibles problemas en la atención de 83 pacientes, como el retraso en el egreso, explicó que $21 \%$ de la estancia hospitalaria de la corte y la mayoría de los casos fueron por retrasos en la provisión de requisitos sociales y terapéuticos. En Colombia, Sánchez HS en el 2017 (15) luego de analizar 27324 observaciones de fallas de calidad en la atención, describen que estas fallas llevaron a una estancia prolongada que generó un sobrecosto de US\$1 380 817. Anotan además, que 7\% de las fallas que explican la estancia inoportuna fueron por problemas asociados con el pagador.

De otro lado, en Colombia las condiciones de salud y la prestación de salud por departamentos, tiene grandes diferencias (16), lo que conlleva a necesidad de traslados interdepartamentales o municipios lejanos a centros que ofrezcan el servicio que requieren los pacientes. Esta situación es vista por algunas entidades como una oportunidad para ofertar sus servicios de mayor complejidad, pero al momento del alta o retornar al sitio de procedencia, esto también pone un reto al sistema de salud, el cual tiene que ofrecer las condiciones adecuadas para el mismo. Sin embargo, el personal del área asistencial observa con relativa frecuencia, que el paciente no puede egresar por diferentes problemas, entre los que se destacan los de tipo administrativo, lo que contribuyen a una estancia inapropiada.

El objetivo de este trabajo fue describir la proporción de pacientes con egreso inoportuno de origen no médico, sus características sociodemográficas, clínicas y estimar los costos directos que de ella se derivaron, durante el periodo comprendido entre el $1^{\circ}$ de junio y el 31 de agosto de 2017 , en una institución de alta complejidad.

\section{Material y métodos}

Se realizó un estudio descriptivo, retrospectivo, de los pacientes que estuvieran hospitalizados y que cumplieran 
con el criterio de egreso inoportuno, entre el $1^{\circ}$ de junio de 2017 y el 31 de agosto del mismo año en la Clínica Somer, la cual es una institución de alta complejidad, ubicada en el municipio de Rionegro, Antioquia. Esta institución cuenta con cerca de 220 camas, con un número de egresos anuales aproximados de 13000 pacientes. La institución presta servicios a entidades del régimen subsidiado, contributivo y particulares, resaltando además que existen convenios de atención para municipios cercanos, pero también para otras regiones del país.

La definición operativa de egreso inoportuno y único criterio de inclusión fue: pacientes que desde el punto de vista clínico (médico tratante) podían ser dados de alta por su condición de salud, pero que continuaban ocupando una cama por problemas no médicos (11). Para obtener los casos, diariamente se registraba por el grupo investigador y el médico del sistema de auditoría de la clínica, los pacientes que cumplieran con este criterio del censo de hospitalización. Posteriormente se verificaba en la historia clínica que cumpliera con el criterio de egreso u orden de alta por el médico tratante y se hacía seguimiento hasta el real momento del alta. Las variables recolectadas fueron: sexo, edad, procedencia, régimen de seguridad social, aseguradora, diagnóstico de egreso, servicio tratante al momento del alta, tiempo total de estancia hospitalaria, días desde el alta médica hasta el egreso de la institución, causa de egreso inoportuno.

Para los costos se tuvieron en cuenta: costo total de la estancia por paciente y el generado por el egreso inoportuno, porcentaje de este costo con respecto al costo total. El análisis se hizo desde la perspectiva del pagador; los costos tenidos en cuenta para el estudio fueron: día de hospitalización (habitación), medicamentos, oxígeno, insumos y evaluaciones médicas. Posteriormente los valores se convirtieron a dólares americanos, utilizando la tasa representativa del mercado promedio para el momento del estudio (2951 pesos por dólar americano). Dado que el alcance del estudio era medir el costo directo total del problema, no se analizó el costo discriminado.

En cuanto al análisis estadístico, para las variables cualitativas, se utilizó frecuencia absoluta y relativa expresada en porcentaje y para las variables cuantitativas, se calculó el promedio con su desviación estándar o mediana con su rango intercuartílico dependiendo de la distribución. Los datos fueron almacenados y procesados en Excel. En cuanto a las consideraciones éticas, se anota que contó con la aprobación del Comité de Ética en investigación de la institución.

\section{Resultados}

Durante los tres meses analizados se encontraron 79 pacientes con egreso inoportuno de origen no médico de un total de 3273 egresos, correspondiente al 2.4\% (Tabla 1). Predominaron las mujeres con $59.5 \%$ de los casos. La mediana para la edad fue de 49 años, con un rango amplio desde seis meses hasta 93 años. La mayoría de los pacientes eran del régimen subsidiado con $62-60.4 \%$ eran procedentes de otros departamentos o municipios lejanos. El total de días de hospitalización de los pacientes fue 1989, con un aporte en días por egreso inoportuno de 547 (correspondió a 27.5\% de la estancia) con un promedio de estancia inoportuna de 6.6 días por paciente.

Las causas de egreso inoportuno identificadas fueron (Figura 1):

- Necesidad de tiquetes para retornar al sitio de procedencia (39.5\%), correspondiente en su mayoría a paciente de bajos recursos, sin posibilidad para cubrir estos gastos.

- Alta temprana con medicamentos (22.8\%) correspondientes a tratamiento generalmente no contemplados en el POS o de programas especiales (anticoagulantes, nutrición, leche complementaria, programa de VIH, TBC, entre otros), sin los cuales el paciente no puede salir de la institución.

- Necesidad de oxígeno domiciliario para el alta (21.5\%)

- Otras causas sumaron 16.5\%: correspondientes a solicitud de ambulancia para el egreso, la cual era necesaria para trasladar pacientes que por su condición crítica no podían realizar viajes en transporte público o incluso privado. Albergues u hogar de paso, indicada como opción para grupos de pacientes cuya procedencia sea lejana y necesitaban terminar un tratamiento, por ejemplo radioterapia, quimioterapia y plan canguro en recién nacidos pretérminos.

Las especialidades más afectadas fueron: medicina interna $24.1 \%$, cuidados paliativos $15.2 \%$, ortopedia $12.6 \%$ y ginecología $11.3 \%$, aunque en términos generales afectó

Tabla 1. Características pacientes con estancia inapropiada, $n=79$.

\begin{tabular}{|l|c|}
\hline Edad mediana en años (rango) & $49(0.5-93)$ \\
\hline Pacientes de sexo femenino, número (\%) & $47(59.5)$ \\
\hline Régimen subsidiado, número (\%) & $49(62)$ \\
\hline $\begin{array}{l}\text { Procedentes de otros departamentos o municipios lejanos, } \\
\text { número (\%) }\end{array}$ & $48(60.7)$ \\
\hline Total, días de egreso inoportuno (rango) & $547(1-25)$ \\
\hline
\end{tabular}

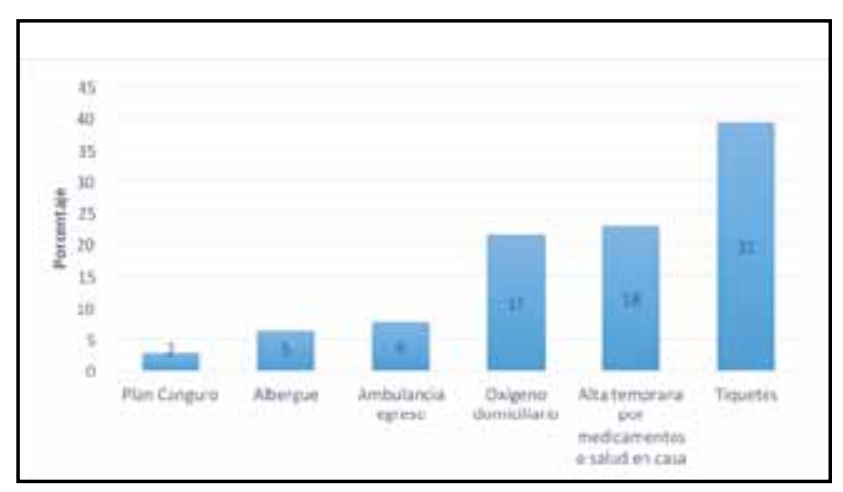

Figura 1. Causas de egreso inoportuno, $n=79$. 
a múltiples áreas de atención, pero en menor proporción (Figura 2). Mencionamos además que los pacientes afectados, ninguno de ellos tuvo un evento adverso durante este periodo.

El costo total de la atención de los 79 pacientes fue US\$637 050.82, de los cuales US\$83 935.66 (13.1\%) correspondió a estancia inoportuna (Tabla 2). Con un valor promedio día de US\$153.44, el cual corresponde a día de hospitalización (habitación), medicamentos, oxígeno, insumos y evaluaciones médicas. El promedio por paciente fue de US\$1062.47. Los servicios más afectados fueron: medicina interna US\$14 709.55, cuidados paliativos US\$11 735.93, pediatría US\$11 383.62 y ortopedia US\$10 123.07, quienes aportaron $57.1 \%$ del costo. Anotamos además que este valor fue asumido por las empresas responsables del pago (ERP). En la Figura 3 se discriminan los costos por servicio de atención.

\section{Discusión}

El egreso inoportuno es un problema en la atención que genera un importante consumo de recursos de forma innecesaria. Podemos ver que en sólo una clínica, durante los tres meses revisados se presentaron 79 casos, que corresponden a $2.4 \%$ del total de egresos. Una revisión sistemática realizada por Landeiro y cols. en el 2017 (3), evidenció que estos casos pueden afectar desde $1.6 \%$ o incluso más de $38 \%$ de todos los pacientes hospitalizados, según la definición del caso que se utilice, población o la institución evaluada. Si bien es un porcentaje bajo, al observar más de cerca vemos que el total de días de hospitalización perdidos por esta causa fue 547, lo que significa el uso de cerca de 18 camas durante un mes en cualquier institución de salud. Esto en una institución de alta complejidad, donde por lo general las camas son un recurso limitado y con otros servicios colapsados esperando que se liberen, es una cifra altamente significativa.

Tabla 2. Distribución de pacientes por mes, costo total y costo por estancia inoportuna, en dólares (1 dólar = 2951 pesos colombianos), año 2017.

\begin{tabular}{|c|c|c|c|c|}
\hline Mes & Pacientes & $\begin{array}{c}\text { Estancia inoportuna } \\
\text { en días }\end{array}$ & $\begin{array}{c}\text { Costo total } \\
\text { de la facturación }\end{array}$ & US\$102 867.81 \\
\hline Junio & 21 & 145 & US\$260 49.31 & US\$22 545.70 (22) \\
\hline Julio & 29 & 184 & US\$273 691.70 & US\$31 $834.26(12)$ \\
\hline Agosto & 29 & 218 & US\$637 050.82 & US\$29 555.70 (11) \\
\hline Total & 79 & 547 & US\$83 935.66 (13.1) \\
\hline
\end{tabular}

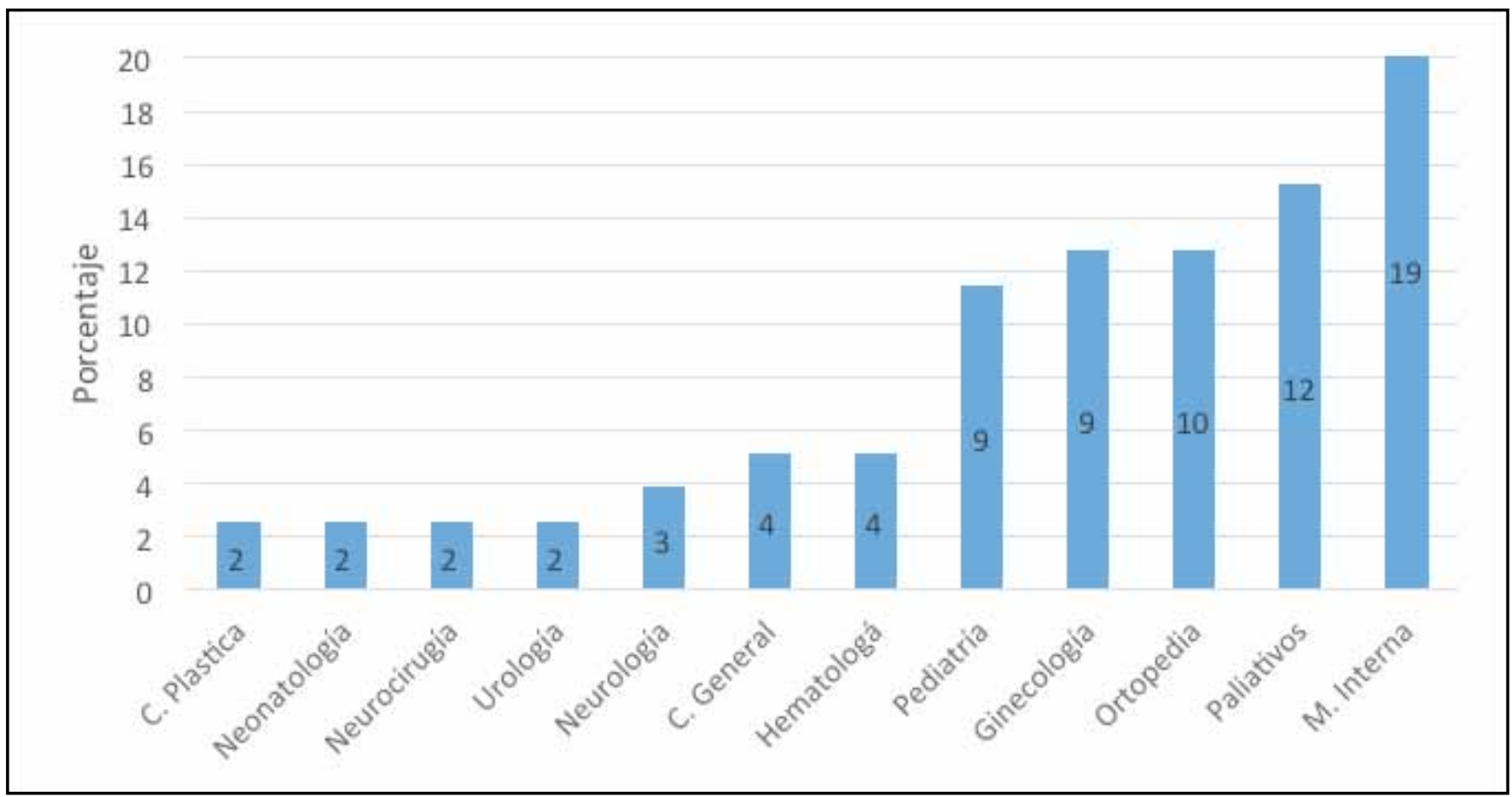

Figura 2. Distribución de causas de egreso inoportuno por servicio, $n=79$. 


\section{$16.000,00$}

\section{$14.000,00$}

$12.000,00$

$10.000,00$

$8.000,00$

$6.000,00$

$4.000,00$

$2.000,00$

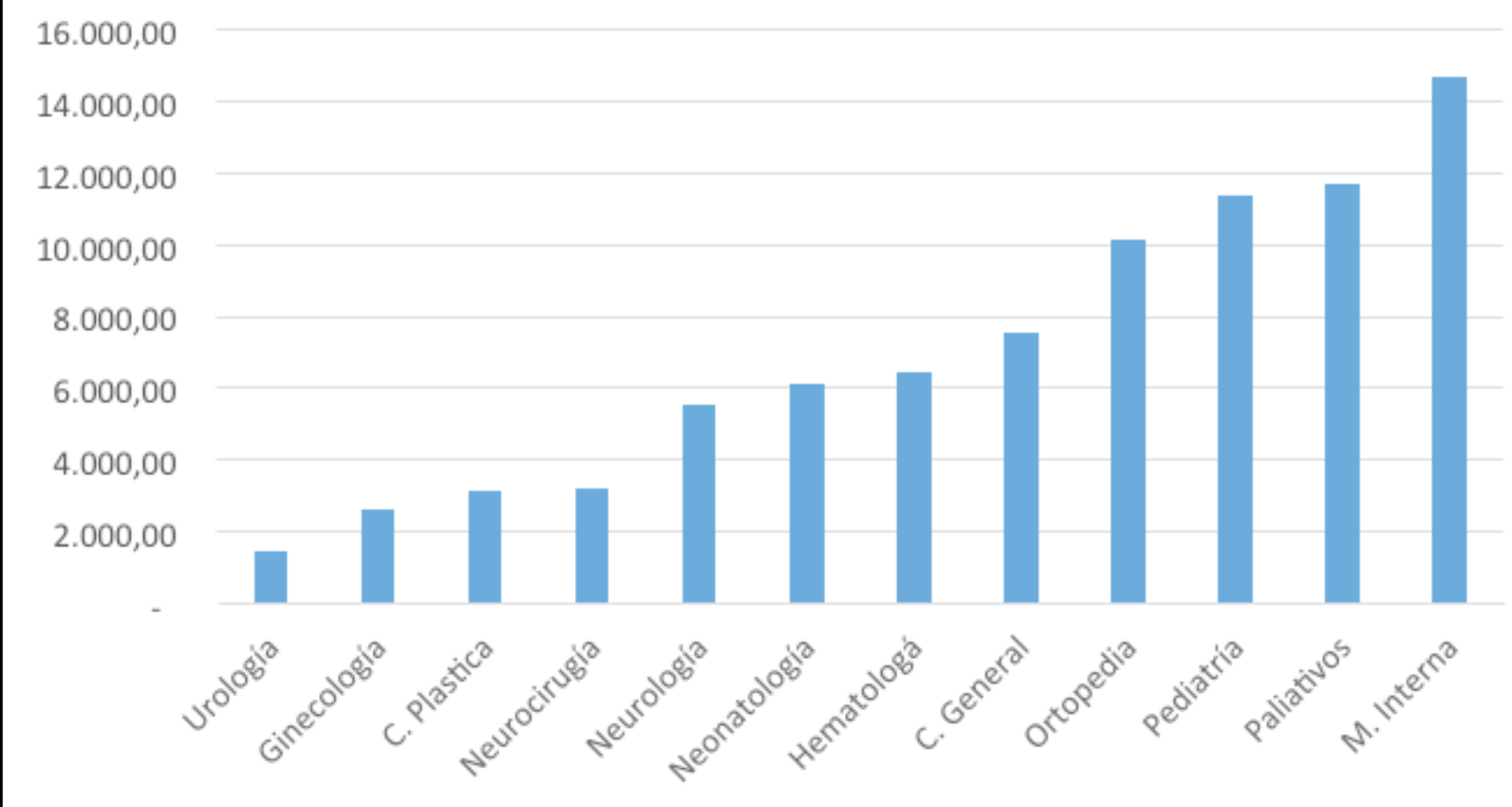

(2)

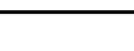

政

2nd

Figura 3. Costos de egreso inoportuno en dólares (1 dólar = 2951 pesos colombianos), por servicio de atención.

En nuestro estudio el egreso inoportuno, estuvo en relación con la procedencia y régimen de salud. Cerca de $60 \%$ de los pacientes corresponden al régimen subsidiado y venían remitidos de otros departamentos o municipios lejanos como San Andrés, Chocó, Santander, occidente Antioqueño, entre otros, y también de comunidades indígenas (datos no mostrados). Es de esperar que esta población sea la más afectada, entendiendo las dificultades geográficas y sociales de nuestro país y donde la red de atención para este régimen puede es más limitada (16) y presenta mayoresbarreras de acceso (17).

El problema afectó a todas las áreas de hospitalización. Los datos analizados, muestran que abarca todas las edades y los servicios de atención de nuestra clínica. Los más afectados fueron medicina interna y cuidados paliativos, posiblemente por ser servicios tratantes o de descarga de otras especialidades, aunque se ha demostrado que la especialidad de medicina interna explica una gran parte de los problemas los retrasos de atención (15). El promedio de días por egreso inoportuno por paciente fue 6.6 días. Otros países como Cuba, México, Reino Unido, han reportado promedios entre 2.2 y 12.7 días $(3,18)$, lo cual depende del tipo de institución o especialidad afectada.

Las principales causas de egreso inoportuno fueron la necesidad de tiquetes para retorno, necesidad de medicamentos para el egreso y necesidad de oxígeno domiciliario con $60 \%$ de los casos. Ese hallazgo concuerda con lo descrito por Sanchez y cols. (15), quienes también encontraron problemas de estancia inoportuna relacionados con la demora de entrega de medicamentos o servicios ambulatorios, oxígeno domiciliario y traslado a hospital en casa. El problema del retorno de los pacientes a su sitio de origen estuvo en relación con la condición social económica baja y un trámite no eficiente en muchos casos por los prestadores de salud, lo que se refleja en una pérdida económica importante. Si bien el traslado domiciliario no está contemplado en nuestro plan obligatorio de salud (POS), gran parte de estos pacientes son de bajos recursos sin la posibilidad de costear sus pasajes. En ocasiones las entidades de salud asumen este gasto, pero mientras se resuelve el alta hospitalaria, los pacientes siguen consumiendo recursos. La necesidad de oxígeno, medicamentos indispensables para el alta y necesidad de ambulancia por la condición clínica del paciente, también reflejan la necesidad de fortalecer los servicios de las aseguradoras.

El egreso inoportuno genera un alto costo para el sistema. El costo generado por los casos encontrados fue US\$83 935.66. El promedio del costo por paciente fue US\$1062.47. Entendemos que esta cifra varía según las diferentes instituciones. Instituciones en Colombia anotan pagos hasta de US\$276 180 por este concepto (15). Datos de otros países muestran que este problema puede costar entre $€ \$ 2352$ y 9317 por pacientes $(13,18)$. En Estados Unidos hay reportes de US $\$ 1.8$ millones por un tiempo similar al de nuestro estudio (19). Lo anterior demuestra que es una cifra muy elevada para el sistema y depende de las características de la institución. Si multiplicamos esta cifra por 12 meses de atención y por todas las instituciones en Colombia, el problema se acrecienta mucho más. Como 
expresamos anteriormente, el pago del costo fue asumido por las empresas responsables del pago (ERP).

\section{Implicaciones del estudio}

La información aportada consideramos que puede ser útil para los administradores de salud, centrándose en la eficiencia del manejo y operatividad de los servicios, en especial para mejorar el proceso de egreso de los pacientes. Es claro que el uso racional de la estancia hospitalaria y ayudas diagnósticas, son elementos claves para la gestión de la calidad y poder así impactar en el desperdicio de recursos. Existen varios actores que deben participar para su solución. Los prestadores de salud deben identificar de forma temprana, los factores que pueden derivarse en un egreso oportuno (incluso desde el ingreso), para intervenirlos. Por ejemplo, un médico puede predecir que su paciente va a requerir oxígeno domiciliario, una nutrición especial o una fórmula complementaria en el caso de un recién nacido para el momento del alta, el paso a seguir es iniciar el trámite de forma temprana.

Las aseguradoras también deben establecer medidas, con el fin de tener una red eficiente de atención, oportunidad en medicamentos, hospital en casa, o definir desde la remisión cómo volverá el paciente a su sitio de origen, sobre todo en pacientes de bajos recursos. Además se debe insistir por los entes territoriales, en mejorar el acceso a servicios de salud de buena calidad y alta complejidad para evitar en lo posible, traslados interdepartamentales.

\section{Áreas de incertidumbre y limitaciones}

Se resalta que los hallazgos de este estudio, se limita a instituciones con características similares. Para entender de forma amplia las implicaciones de los egresos inoportunos, se debe observar el comportamiento del problema a nivel particular y en diferentes niveles de atención. Anotamos también que el tiempo evaluado fue corto, debido por el carácter exploratorio del estudio, pero es conveniente analizar el problema en periodos más largos para evitar sesgo de periodos y ver el comportamiento en el tiempo.

No fue del alcance del estudio medir los costos o pérdidas que conlleva a la institución ni los costos que genera esta estancia para los pacientes, lo cual también sería un área para investigar.

\section{Referencias}

1. Expert I. Independent Expert Review of of Delayed Delayed Discharges. 2018;(September).

2. Caminiti C, Meschi T, Braglia L, Diodati F, Iezzi E, Marcomini B, et al. Reducing unnecessary hospital days to improve quality of care through physician accountability: a cluster randomised trial. BMC Health Serv Res [Internet].
2013 [citado 10 de abril de 2018];13. Disponible en: http://www.biomedcentral. com/1472-6963/13/14

3. Landeiro F, Roberts K, Gray AM, Leal J. Delayed Hospital Discharges of Older Patients: A Systematic Review on Prevalence and Costs. 2019;59(2):86-97.

4. Tudela P, Mòdol JM. La saturación en los servicios de urgencias hospitalarios. [citado 2 de mayo de 2018]; Disponible en: http://formacionemergencias.portalsemes.org/formacion/pdf/2015_1_semestre/saturacion_servicios_urgencias_hospitalarios.pdf

5. Fontana E, Rosen A, Shkuda V, Walsh L, Woodburn S. Next-Generation Capacity Management. 2010;

6. Social MDLP. Protocolo de revisión de la utilización de la estancia hospitalaria (PRU) [Internet]. 2009. Disponible en: https://www.minsalud.gov.co/sites/rid/ Lists/BibliotecaDigital/RIDE/DE/CA/Eficiencia_con_calidad.pdf

7. María Ceballos-Acevedo T, Andrea Velásquez-Restrepo P, Sebastián JaénPosada J, Universitaria I, Los Alpes B. Duración de la estancia hospitalaria. Metodologías para su intervención * Length of the Hospitalization. Methodologies for Intervention Duração da estancia hospitalar. Metodologias para sua intervenção. Rev Gerenc Polít Salud Rev Gerenc Polit Salud [Internet]. 2014 [citado 10 de abril de 2018];13(1327):274-95. Disponible en: http://dx.doi.

8. Javier Luquero Alcalde F, Santos Sanz S, Pérez Rubio A, Tamames Gómez S, Belén Cantón Álvarez M, Castrodeza Sanz J. Factores determinantes de la estancia inadecuada en un hospital de tercer nivel. Gac Sanit [Internet]. 2008 [citado 27 de abril de 2018];22(1):48-51. Disponible en: https://ac.els-cdn.com/ S0213911108712019/1-s2.0-S0213911108712019-main.pdf?_tid=1d16107d543d-4df5-9f90-59212d9ca367\&acdnat=1524855862_eda98e1101fb07de4b$9 f 6 e=9 \mathrm{a} 0 \mathrm{a} 3 \mathrm{c} 6 \mathrm{~cd}$

9. Fernanda Henao Obando Madeleyne Jaramillo Zapata Asesor José Humberto Duque Zea L, Javeriana P, Fernanda Henao Obando Madeleyne Jaramillo Zapata L, José Humberto Duque Zea A. Evaluación de la adecuación de la estancia hospitalaria, en una clínica privada de Medelín de mediana y alta complejidad [Internet]. 2011 [citado 27 de abril de 2018]. Disponible en: http://bdigital.ces. edu.co:8080/jspui/bitstream/10946/573/1/Evaluacion_adecuacion_estancia.pdf

10. Michael J, Emrys HS. Article : Delayed Discharges and Hospital Type : Evidence from the English NHS *. 2017;

11. Mendoza Giraldo D, Navarro A, Sánchez-Quijano A, Villegas A, Asencio R, Lissen E. Retraso del alta hospitalaria por motivos no medicos. Rev Clin Esp. 2012;212(5):229-34.

12. Kim CS, Hart AL, Paretti RF, Kuhn L, Dowling AE, Benkeser JL, et al. Excess hospitalization days in an academic medical center: Perceptions of hospitalists and discharge planners. Am J Manag Care. 2011;17(2):34-42.

13. Rojas-García A, Turner S, Pizzo E, Hudson E, Thomas MMus GGSM JM, Raine MBBS R, et al. Impact and experiences of delayed discharge: A mixedstudies systematic review. Heal Expect [Internet]. 2018 [citado 2 de mayo de 2018];21:41-56. Disponible en: https://www.ncbi.nlm.nih.gov/pmc/articles/ PMC5750749/pdf/HEX-21-41.pdf

14. Hendy P, Patel JH, Kordbacheh T, Laskar N, Harbord M. In-depth analysis of delays to patient discharge : a metropolitan teaching hospital experience. 2012;12(4):320-3.

15. Carlos HS. Costos financieros de la baja calidad por aumento de la estancia hospitalaria. 2017;5(7):55-62.

16. Ministerio de Salud y Protección Social, Dirección de Epidemiología y Demografía. Análisis de situación de salud según regiones Colombia [Internet]. [citado 27 de abril de 2018]. Disponible en: https://www.minsalud.gov.co/Documentos y Publicaciones/Análisis de situación de salud por regiones.pdf

17. Del A, Agudelo C. Barreras de acceso a los servicios de salud en el régimen subsidiado del municipio de San Gil - Santander (tesis de especialización en generencia de la seguridad social). Universidad autónoma de Bucaramanga. 2013; disponible en: http://bdigital.ces.edu.co:8080/repositorio/bitstream/10946/1841/2/ BARRERAS_ACCESO_SERVICIOS_SALUD_SAN_GIL.pdf

18. Mould-quevedo JF, García-peña C, Contreras- I, Juárez-cedillo T, Espinelbermúdez $\mathbf{C}$, Morales-cisneros $\mathbf{G}$, et al. Direct costs associated with the appropriateness of hospital stay in elderly population. 2009;8:1-8.

19. Jr ML, Bachim A, Smith C, Camp EA, Donaruma-kwoh M, Patel B. Hospital Costs and Charges of Discharge Delays in Children Hospitalized for Abuse and Neglect. 2019. 